\title{
A Miniaturized Fluorescence Detector with a Windowless Flow Cell Using a Light-Emitting Diode
}

\author{
Bingcheng YANG and Yafeng GUAN ${ }^{\dagger}$ \\ Dalian Institute of Chemical Physics, Chinese Academy of Sciences, 116012, P. R. China
}

\begin{abstract}
A miniaturized fluorescence detector using a high-brightness light-emitting diode as an excitation source was constructed and evaluated. A windowless flow cell based on a commercial four-port cross fitting was designed to reduce the straylight level and to eliminate the optical alignment. The observed detection limit for fluorescein was $26 \mathrm{nM}$ in the continuous-flow mode. The error in the reproducibility of the responses was evaluated by the FIA method, and was found to be within $2 \%$ RSD.
\end{abstract}

(Received October 28, 2002; Accepted December 13, 2002)

\section{Introduction}

The direction of miniaturization of analytical devices may be a main trend during the present decade..$^{1,2}$ One of the challenges in the development of miniaturized analytical devices is the miniaturization of detectors. Fluorescence $(\mathrm{Fl})$ detection is frequently applied in microscale separation techniques because of its high sensitivity. The use of lasers and lamps has been demonstrated as excitation sources for miniaturized fluorescence instruments. ${ }^{2,3}$ Common limitations result from bulky size and high power consumption. The use of light emitting diodes (LEDs) as excitation sources may provide a simple alternative., ${ }^{4,5}$ However, due to the small injection volume and flow channel, it is necessary to make the volume of the flow cell compatible with the sample peak in order to avoid sample dispersion. The most popular approach to achieving a low detection volume is on-column detection. In this arrangement, due to the cylindrical section and other adverse factors, it results in a high stray-light level. In addition, there exits an inherent difficulty for optical alignment in on-column detection.

Here, we present a novel Fl detector design, which uses a commercial four-port cross fitting as the flow cell and a blue high-brightness LED as the excitation source. The performance of the detector was evaluated using a fluorescein dye in the continuous-flow mode, and its suitability was demonstrated by a flow injection analysis (FIA) method.

\section{Experimental}

\section{Apparatus and chemicals}

The light emanating from a blue LED (peak wavelength, 470 $\mathrm{nm}$; luminous power, $2 \mathrm{~mW}$; Shifeng Optic and Electronics Ltd., China) was collimated and focused with two quartz lenses (Dahen Optic and Electronics Ltd., China) into a fiber (core, $200 \mu \mathrm{m}$; cladding, $220 \mu \mathrm{m}$; Chunhui, China). The fibers carried

† To whom correspondence should be addressed.

E-mail: guan_yafeng@yahoo.com.cn; kfguan@mail.dlptt.ln.cn the excitation light beam into the flow cell and collected the Fl with a right-angle geometry. The Fl then passed through two blocks of an interference band pass filter (peak wavelength, 530 nm; FWHM, 30 nm; Huibo, China), and was then detected by a side-on photomultiplier tube (PMT) (Type R928, Hamamatsu). The output signal was recorded with an HP-3395 integrator or a strip-chart recorder. The obtained power of the excitation light at the output end of the fiber was approximately $160 \mu \mathrm{W}$. A homemade enclosure shielded the optical assembly from external light.

Fluorescein was obtained from Sigma (USA) and deionized (DI) water was used throughout.

\section{Construction of the windowless flow cell}

According to the Fresnel formula, the larger is the difference of the refractive index (RI) at the two-phase interfaces, the greater is the level of stray light due to the refraction and reflection of the optical beam. In fact, the stray light at the interfaces is more pronounced when they are not flat, but have a curvature, as in the case of capillaries. To eliminate stray light from the light window, some novel designs of flow cell with no wall at the detection portion, known as windowless flow cell, have been presented. ${ }^{6-8}$ Hackett et al. used a commercial fourport cross fitting as a windowless flow cell for absorption detection. $^{6}$ We modified the design so as to apply the $\mathrm{Fl}$ detection system, as shown in Fig. 1. In this design, an optical fiber carried the excitation beam to the flow cell, and the emitted fluorescent light from the sample was transferred to a PMT by another fiber located perpendicular to the first fiber. The inlet and outlet capillaries were fixed at two other ports of the cross fitting, respectively. The fibers were in physical contact with the analyte stream, which reduced the stray light due to the smaller RI difference among them. PTFE tubing $(0.25 \mathrm{~mm}$ i.d. $\times 1.6 \mathrm{~mm}$ o.d. $)$ was used to seal the fibers and the capillaries. The PTFE tubing was drilled along the axis with micro-aiguilles to fit with the outer diameters of the capillaries or the fibers. The good precision of the commercial cross fitting obviated any need for an additional optical alignment among the capillaries and the fibers. The total cell volume was about 20 $\mathrm{nL}$. 


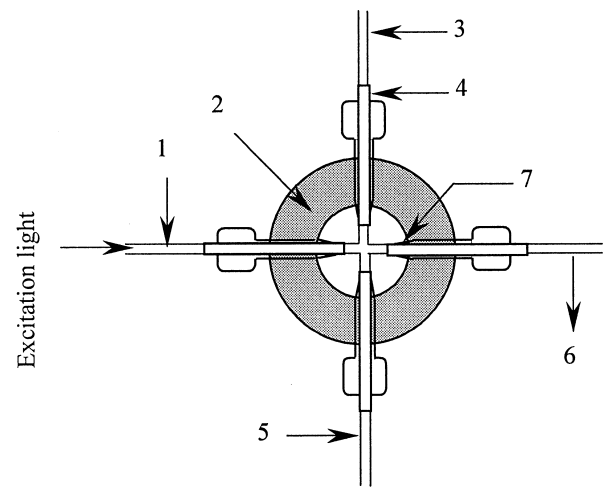

Fig. 1 Schematic of the cross flow cell. 1 and 3, optical fiber; 2, flow cell; 4, PTFE tube; 5, inlet capillary; 6, outlet capillary; 7, ferrule.

\section{Results and Discussion}

For a comparison, an Fl detector based on the on-column mode was assembled and the cross cell was replaced by a capillary with $0.25 \mathrm{~mm}$ i.d.; the other conditions remained constant. The noise level was about $8 \mu \mathrm{V}$ and $30 \mu \mathrm{V}$ (peak-to-peak) for the detector and the on-column detector, respectively. The drift was about $40 \mu \mathrm{V} / 30 \mathrm{~min}$ for both detector systems. The signalto-noise ratio for continuous pumping of $5 \mu \mathrm{M}$ fluorescein through the capillary was 575. Thus, assuming linearity, the concentration detection limit, defined as 3-times the baseline noise, was $26 \mathrm{nM}$, which is much lower than that of the oncolumn detector, $140 \mathrm{nM}$. The reproducibility of the system is illustrated by Fig. 2, showing 6 replicate sample injections using the FIA technique. The RSD value was $2 \%$. Although the obtained detection limit was much higher than those usually achieved by laser-induced Fl detection, ${ }^{9}$ this detector was simple, cheap and robust. Further improvements in detectability could be obtained by the use of an LED powered by the pulsed mode.

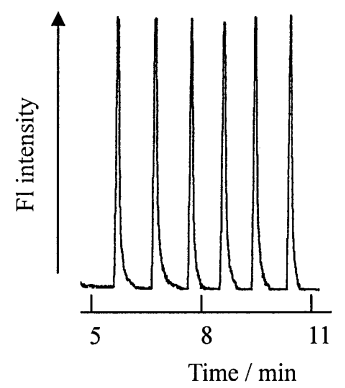

Fig. 26 replicate injections of the reproducibility of $5 \mu \mathrm{M}$ fluorescein. Carrier rate, $40 \mu \mathrm{L} / \mathrm{min}$; carrier tube, $0.25 \mathrm{~mm}$ i.d; injection volume, $500 \mathrm{~nL}$; PMT voltage, $800 \mathrm{~V}$.

\section{Acknowledgements}

This work was supported by the National Natural Science Foundation of China (No. 29925514).

\section{References}

1. K. Kuruma and T. Sakano, Anal. Sci., 1999, 15, 489.

2. X.-Z. Wu and J. Pawliszyn, Electrophoresis, 1995, 16, 1474.

3. T. Kaneta, T. Komatsubara, H. Shiba, and T. Imasaka, Anal. Sci., 1998, 14, 1017.

4. M. Macka, P. Andersson, and P. R. Haddad, Electrophoresis, 1996, 17, 1898.

5. H. Liu, P. K. Dasgupta, and H. J. Zheng, Talanta, 1993, 40, 1331.

6. H. Wang, E. C. Yi, C. A. Ibarra, and M. Hackett, Analyst [London], 2000, 125, 1061.

7. G. J. Diebold and R. N. Zare, Science, 1977, 196, 1439.

8. G. L. Klein, U. S. Patent, 1994, 5302272; Chem. Abstr., 1994, 120, 293558j.

9. T. Anazawa, S. Takahashi, and H. Kambara, Electrophoresis, 1999, 20, 539. 